\title{
On the eigenvalues of Euclidean distance matrices
}

\author{
A.Y. ALFAKIH* \\ Department of Mathematics and Statistics \\ University of Windsor, Windsor, Ontario N9B 3P4, Canada \\ E-mail: alfakih@uwindsor.ca
}

\begin{abstract}
In this paper, the notion of equitable partitions (EP) is used to study the eigenvalues of Euclidean distance matrices (EDMs). In particular, EP is used to obtain the characteristic polynomials of regular EDMs and non-spherical centrally symmetric EDMs. The paper also presents methods for constructing cospectral EDMs and EDMs with exactly three distinct eigenvalues.
\end{abstract}

Mathematical subject classification: 51K05, 15A18, 05C 50.

Key words: Euclidean distance matrices, eigenvalues, equitable partitions, characteristic polynomial.

\section{Introduction}

An $n \times n$ nonzero matrix $D=\left(d_{i j}\right)$ is called a Euclidean distance matrix (EDM) if there exist points $p^{1}, p^{2}, \ldots, p^{n}$ in some Euclidean space $\Re^{r}$ such that

$$
d_{i j}=\left\|p^{i}-p^{j}\right\|^{2} \quad \text { for all } i, j=1, \ldots, n,
$$

where \|\| denotes the Euclidean norm.

Let $p^{i}, i \in N=\{1,2, \ldots, n\}$, be the set of points that generate an EDM $D$. An m-partition $\pi$ of $D$ is an ordered sequence $\pi=\left(N_{1}, N_{2}, \ldots, N_{m}\right)$ of nonempty disjoint subsets of $N$ whose union is $N$. The subsets $N_{1}, \ldots, N_{m}$ are called the cells of the partition. The $n$-partition of $D$ where each cell consists

\#760/08. Received: 07/IV/08. Accepted: 17/VI/08.

* Research supported by the Natural Sciences and Engineering Research Council of Canada and MITACS. 
of a single point is called the discrete partition, while the 1-partition of $D$ with only one cell is called the single-cell partition.

An $m$-partition $\pi=\left(N_{1}, N_{2}, \ldots, N_{m}\right)$ of an EDM $D$ is said to be equitable if for all $i, j=1, \ldots, m$ (case $i=j$ included), there exist non-negative scalars $\alpha_{i j}$ such that for each $k \in N_{i}$, the sum of the squared Euclidean distances from $p^{k}$ to all points $p^{l}, l$ in $N_{j}$, is equal to $\alpha_{i j}$. i.e.,

$$
\forall k \in N_{i}, \quad \sum_{l \in N_{j}} d_{k l}=\alpha_{i j}, \text { for all } i, j=1, \ldots, m .
$$

The notion of equitable partitions for graphs, which was introduced by Sachs [13], is related to, among others, automorphism groups of graphs and distanceregular graphs [4]. Schwenk [15] used equitable partitions to find the eigenvalues of the adjacency matrix of a graph. Hayden et al. [7] also used equitable partitions, albeit under the name block structure, to investigate EDMs generated by points lying on a collection of concentric spheres. In particular, they devised an algorithm for finding the least number of concentric spheres containing the points that generate a given EDM. Their investigation was based on the block structure of EDMs and the corresponding eigenvectors.

Many of the results on the spectra of graphs obtained using equitable partitions have analogous counterparts in the case of EDMs. In particular, we show (see Theorem 3.1) that the characteristic polynomial of an EDM can be written as the product of the characteristic polynomials of two matrices associated with partitions. Theorem 3.1 is then used to determine the characteristic polynomials of regular EDMs and non-spherical centrally symmetric EDMs. We also present methods for constructing cospectral EDMs and non-regular EDMs with exactly three distinct eigenvalues.

Recently, EDMs have received a great deal of attention for their many important applications. These applications include, among others, molecular conformation problems in chemistry [2], multidimensional scaling in statistics [9], and wireless sensor network localization problems [16].

We denote the identity matrix of order $n$ by $I_{n}$ and the $n$-vector of all 1 's by $e_{n} . \quad E_{n, m}=e_{n} e_{m}^{T}$ denotes the $n \times m$ matrix of all 1's, and $E_{n}=e_{n} e_{n}^{T}$ denotes the square matrix of order $n$ of all 1 's. The subscripts of $I, e$ and $E$ will be deleted if the order is clear from the context. For a matrix $A, \operatorname{diag} A$ 
denotes the vector consisting of the diagonal entries of $A$. Finally, the spectrum of a matrix $A$, denoted by $\sigma(A)$, is the multiset of the eigenvalues of $A$. If $A$ has eigenvalues $\lambda_{1}, \ldots, \lambda_{k}$ with multiplicities $m_{1}, \ldots, m_{k}$ respectively, then $\sigma(D)=\left\{\lambda_{1}^{m_{1}}, \ldots, \lambda_{k}^{m_{k}}\right\}$.

\section{Preliminaries}

Let $D$ be an $n \times n$ EDM, the dimension of the affine span of the points $p^{1}, \ldots, p^{n}$ that generate $D$ is called the embedding dimension of $D$. Let $J_{n}:=I_{n}-e_{n} e_{n}^{T} / n$ denote the orthogonal projection on subspace

$$
M:=e^{\perp}=\left\{x \in \Re^{n}: e^{T} x=0\right\} .
$$

It is well known $[14,18]$ that a symmetric matrix $D$ with zero diagonal is an EDM if and only if $D$ is negative semidefinite on $M$. Hence, EDMs have exactly one positive eigenvalue. Let $S_{H}$ denote the subset of $n \times n$ symmetric matrices with zero diagonal; and let $S_{C}$ denote the subset of $n \times n$ symmetric matrices $A$ satisfying $A e=0$. Following [3], let $\mathcal{T}: S_{H} \rightarrow S_{C}$ and $\mathcal{K}: S_{C} \rightarrow S_{H}$ be the two linear maps defined by

$$
\mathcal{T}(D):=-\frac{1}{2} J D J
$$

and

$$
\mathcal{K}(B):=\operatorname{diag}(B) e^{T}+e(\operatorname{diag}(B))^{T}-2 B .
$$

It, then, immediately follows [3] that $\mathcal{T}$ and $\mathcal{K}$ are mutually inverse, and that $D$ in $S_{H}$ is an EDM of embedding dimension $r$ if and only if $\mathcal{T}(D)$ is positive semidefinite of rank $r$.

Let $D$ be an $n \times n$ EDM of embedding dimension $r$ generated by the points $p^{1}, \ldots, p^{n}$ in $\Re^{r}$. Then the $n \times r$ matrix

$$
P=\left[\begin{array}{c}
p^{1^{T}} \\
\vdots \\
p^{n T}
\end{array}\right]
$$

is called a realization of $D$. Given an EDM $D$, a realization $P$ of $D$ can be obtained by factorizing $\mathcal{T}(D)$ into $\mathcal{T}(D)=P P^{T}$. Note that if $P$ is a realization 
of $D$, then $P^{\prime}=P Q$ is also a realization of $D$ for any $r \times r$ orthogonal matrix $Q$. Obviously, $P$ and $P^{\prime}$ in this case are obtained from each other by a rigid motion such as a rotation or a translation.

An EDM $D$ is said to be spherical if the points that generate $D$ lie on a hypersphere, otherwise, it is said to be non-spherical. It is well known $[6,17]$ that an EDM $D$ of embedding dimension $r$ is spherical if and only if rank $D=r+1$, and that $D$ is non-spherical if and only if rank $D=r+2$. A spherical EDM $D$ is said to be regular if the points $p^{1}, \ldots, p^{n}$ that generate $D$ lie on a hyper-sphere whose center coincides with the centroid of $p^{1}, \ldots, p^{n}$. It is not difficult to show that $[12,8]$ an EDM $D$ is regular if and only if $e$ is an eigenvector of $D$ corresponding to the eigenvalue $\frac{1}{n} e^{T} D e$.

\section{Equitable partition for EDMs}

It immediately follows from (1) that the discrete partition of $D$ is always equitable with $\alpha_{i j}=d_{i j}$; while the single-cell partition of $D$ is equitable if and only if $D$ is regular. In the latter case, $\alpha_{11}=\frac{1}{n} e^{T} D e$. It also follows from (1) that

$$
\forall i=1, \ldots, m \text { and } \forall k \in N_{i} \text { we have }(D e)_{k}=\sum_{j=1}^{m} \alpha_{i j} .
$$

Let $\pi=\left(N_{1}, N_{2}, \ldots, N_{m}\right)$ be an $m$-partition of an $n \times n$ EDM $D$ where $\left|N_{i}\right|=n_{i}$ for $i=1, \ldots, m$. Define the $n \times m$ matrix $P_{\pi}=\left(p_{i j}\right)$ such that

$$
p_{i j}=\left\{\begin{array}{cl}
n_{j}^{-1 / 2} & \text { if } i \in N_{j} \\
0 & \text { otherwise. }
\end{array}\right.
$$

$P_{\pi}$ is called the normalized characteristic matrix [5] of $\pi$ since its $j$ th column is equal to $n_{j}^{-1 / 2}$ times the characteristic vector of $N_{j}$, and since $P_{\pi}^{T} P_{\pi}=I_{m}$.

Next we present a lemma whose graph adjacency matrix counterpart was proved by Godsil and McKay [5].

Lemma 3.1. Let $\pi$ be an m-partition of an EDM D. Then $\pi$ is equitable if and only if there exists an $m \times m$ symmetric matrix $S=\left(s_{i j}\right)$ such that

$$
D P_{\pi}=P_{\pi} S
$$

Furthermore, if $\pi$ is an equitable partition then $s_{i j}=\left(n_{i} / n_{j}\right)^{1 / 2} \alpha_{i j}$. 
Proof. Assume that $\pi$ is equitable then for all $k \in N_{i}$ and for all $j=1, \ldots, m$ we have

$$
\left(D P_{\pi}\right)_{k j}=n_{j}^{-1 / 2} \sum_{l \in N_{j}} d_{k l}=n_{j}^{-1 / 2} \alpha_{i j}=\left(n_{i}\right)^{-1 / 2}\left(\frac{n_{i}}{n_{j}}\right)^{1 / 2} \alpha_{i j}=\left(P_{\pi} S\right)_{k j},
$$

where $s_{i j}=\left(n_{i} / n_{j}\right)^{1 / 2} \alpha_{i j}$.

On the other hand, assume that (7) holds for some partition $\pi$. Then for all $k \in N_{i}$ and all $j=1, \ldots, m$, we have

$$
\left(D P_{\pi}\right)_{k j}=n_{j}^{-1 / 2} \sum_{l \in N_{j}} d_{k l}=\left(P_{\pi} S\right)_{k j}=\left(n_{i}\right)^{-1 / 2} s_{i j} .
$$

Therefore, $\sum_{l \in N_{j}} d_{k l}=\left(n_{j} / n_{i}\right)^{1 / 2} s_{i j}$, which is independent of $k$. Hence $\pi$ is equitable.

Given an $m$-partition $\pi$ of EDM $D$ with $m \leq n-1$, let $\bar{P}_{\pi}$ be the $n \times(n-$ m) matrix such that $\left[\begin{array}{ll}P_{\pi} & \bar{P}_{\pi}\end{array}\right]$ is an orthogonal matrix. Let $\chi_{A}(\lambda)$ denote the characteristic polynomial of matrix $A$. Then we have the following two results:

Theorem 3.1. Let $\pi$ be an equitable m-partition of an $n \times n E D M D$, where $m \leq n-1$. Then

$$
\chi_{D}(\lambda)=\chi_{S}(\lambda) \chi_{\bar{S}}(\lambda)
$$

where $S$ is defined in (7) and $\bar{S}=\bar{P}_{\pi}^{T} D \bar{P}_{\pi}$.

Proof. It follows from (7) and the definition of $\bar{P}_{\pi}$ that $P_{\pi}^{T} D \bar{P}_{\pi}=S P_{\pi}^{T} \bar{P}_{\pi}=0$. Thus,

$\operatorname{det}\left(\lambda I_{n}-\left[\begin{array}{c}P_{\pi}^{T} \\ \bar{P}_{\pi}^{T}\end{array}\right] D\left[P_{\pi} \bar{P}_{\pi}\right]\right)=\operatorname{det}\left[\begin{array}{cc}\lambda I_{m}-P_{\pi}^{T} D P_{\pi} & 0 \\ 0 & \lambda I_{n-m}-\bar{P}_{\pi}^{T} D \bar{P}_{\pi}\end{array}\right]$.

Hence,

$$
\operatorname{det}\left(\lambda I_{n}-D\right)=\operatorname{det}\left(\lambda I_{m}-S\right) \operatorname{det}\left(\lambda I_{n-m}-\bar{S}\right) .
$$

Note that in case of discrete partitions, i.e., in case $m=n$, we have $S=D$ thus $\chi_{D}(\lambda)=\chi_{S}(\lambda)$ follows trivially. An analogous result for graphs, namely that the characteristic polynomial of $S$ divides the characteristic polynomial of a graph was obtained by Mowshowitz [10], and by Schwenk [15]. 
Theorem 3.2. Let $\pi$ be an equitable m-partition of an $n \times n E D M D$, where $m \leq n-1$. Then

$$
\chi_{\bar{S}}(\lambda) \text { divides } \chi_{-2 \mathcal{T}(D)}(\lambda),
$$

where $\bar{S}$ is as defined in Theorem 3.1 .

Proof. It follows from (5) and the definition of $\bar{P}_{\pi}$ that $\bar{P}_{\pi}^{T} D e=0$ and $\bar{P}_{\pi}^{T} e=0$. Thus,

$$
\bar{P}_{\pi}^{T} \mathcal{T}(D) P_{\pi}=0 \text { and } \bar{P}_{\pi}^{T} \mathcal{T}(D) \bar{P}_{\pi}=-\frac{1}{2} \bar{P}_{\pi}^{T} D \bar{P}_{\pi}=-\frac{\bar{S}}{2} .
$$

Hence,

$$
\begin{gathered}
\operatorname{det}\left(\lambda I_{n}+2\left[\begin{array}{c}
P_{\pi}^{T} \\
\bar{P}_{\pi}^{T}
\end{array}\right] \mathcal{T}(D)\left[P_{\pi} \bar{P}_{\pi}\right]\right) \\
=\operatorname{det}\left[\begin{array}{cc}
\lambda I_{m}+2 P_{\pi}^{T} \mathcal{T}(D) P_{\pi} & 0 \\
0 & \lambda I_{n-m}+2 \bar{P}_{\pi}^{T} \mathcal{T}(D) \bar{P}_{\pi}
\end{array}\right] .
\end{gathered}
$$

Therefore,

$$
\operatorname{det}\left(\lambda I_{n}+2 \mathcal{T}(D)\right)=\operatorname{det}\left(\lambda I_{m}+2 P_{\pi}^{T} \mathcal{T}(D) P_{\pi}\right) \operatorname{det}\left(\lambda I_{n-m}-\bar{S}\right) .
$$

\section{Applications of Theorem 3.1}

In this section we show that Theorem 3.1 provides a new method for determining the characteristic polynomials of regular EDMs and non-spherical centrally symmetric EDMs. It also provides a method for constructing cospectral EDMs.

Let $V_{n}$ be the $n \times(n-1)$ whose columns form an orthonormal basis for subspace $M$ defined in (2), i.e., $V_{n}$ has full column rank and satisfies

$$
V_{n}^{T} e_{n}=0, \quad V_{n}^{T} V_{n}=I_{n-1}, \quad V_{n} V_{n}^{T}=J_{n}=\left(I_{n}-e_{n} e_{n}^{T} / n\right) .
$$

\section{$4.1 \chi_{D}(\lambda)$ of regular EDMs}

The characteristic polynomial of a regular EDM was obtained in $[8,1]$. The method used in [1] is based on a characterization of the nullspace of an EDM in terms of its Gale subspace. Next we determine the characteristic polynomial of regular EDMs as a corollary of Theorem 3.1. 
Corollary $4.1([8, \mathbf{1}])$. Let $D \neq 0$ be an $n \times n$ regular EDM of embedding dimension $r$, then

$$
\chi_{D}(\lambda)=\lambda^{n-r-1}\left(\lambda-\frac{1}{n} e^{T} D e\right) \prod_{i=1}^{r}\left(\lambda+2 \mu_{i}\right),
$$

where $\mu_{i}$, for $i=1, \ldots, r$, are the nonzero eigenvalues of $\mathcal{T}(D)$.

Proof. Let $D$ be an $n \times n$ regular EDM. Then, as we remarked earlier, the onecell partition $\pi$ of $D$, in this case, is equitable. Since $P_{\pi}=\frac{1}{\sqrt{n}} e$ and $\bar{P}_{\pi}=V_{n}$ we have $S=\frac{1}{n} e^{T} D e$ and $\bar{S}=V_{n}^{T} D V_{n}=-2 V_{n}^{T} \mathcal{T}(D) V_{n}$. Thus,

$$
\chi_{S}(\lambda)=\lambda-\frac{1}{n} e^{T} D e \text { and } \chi_{\bar{S}}(\lambda)=\lambda^{n-r-1} \prod_{i=1}^{r}\left(\lambda+2 \mu_{i}\right)
$$

since the nonzero eigenvalues of $\bar{S}$ are equal to the nonzero eigenvalues of the matrix $-2 \mathcal{T}(D)$.

\section{$4.2 \chi_{D}(\lambda)$ of non-spherical centrally symmetric EDMs}

Let $D_{1}$ be the $2 n \times 2 n$ EDM generated by the points $p^{1}, \ldots, p^{n}, \ldots, p^{2 n}$, where $p^{n+i}=-p^{i}$ for all $i=1, \ldots, n$. Assume that $D_{1}$ is non-spherical. Then $D_{1}$ is called a non-spherical centrally symmetric EDM. It is easy to see that

$$
D_{1}=\left[\begin{array}{cc}
D & A \\
A & D
\end{array}\right]
$$

where $D$ is the $n \times n$ EDM generated by the points $p^{1}, \ldots, p^{n}$, and

$$
A=\operatorname{diag}(\mathcal{T}(D)) e_{n}^{T}+e_{n} \operatorname{diag}(\mathcal{T}(D))^{T}+2 \mathcal{T}(D)
$$

The characteristic polynomial of $D_{1}$, which was obtained in [1] using a characterization of the nullspace of an EDM in terms of its Gale subspace, is given by

$$
\chi_{D_{1}}(\lambda)=\lambda^{2 n-r-2}\left(\lambda^{2}-\lambda \frac{1}{2 n} e_{2 n}^{T} D_{1} e_{2 n}-\frac{2 n}{x_{1}^{T} x_{1}}\right) \prod_{i=1}^{r}\left(\lambda+2 \mu_{i}\right),
$$


where $r$ is the embedding dimension of $D_{1}, \mu_{i}, i=1, \ldots, r$, are the nonzero eigenvalues of $\mathcal{T}\left(D_{1}\right)$, and $x_{1} \in \mathfrak{R}^{2 n}$ is the unique vector satisfying

$$
D_{1} x_{1}=e_{2 n}, \quad e_{2 n}^{T} x_{1}=0, \quad \text { and } x_{1} \perp \text { Nullspace of } D_{1} .
$$

Note that such $x_{1}$ satisfying (13) exists since $D_{1}$ is non-spherical. A simple method to compute $x_{1}$ is given in [1]. Next, we establish (12) using Theorem 3.1.

The $n$-partition $\pi$ of $D_{1}$ corresponding to the normalized characteristic matrix

$$
P_{\pi}=\frac{1}{\sqrt{2}}\left[\begin{array}{c}
I_{n} \\
I_{n}
\end{array}\right]
$$

is, obviously, equitable. Since

$$
\bar{P}_{\pi}=\frac{1}{\sqrt{2}}\left[\begin{array}{r}
I_{n} \\
-I_{n}
\end{array}\right]
$$

it immediately follows that $S=P_{\pi}^{T} D_{1} P_{\pi}=D+A=2\left(\operatorname{diag}(\mathcal{T}(D)) e_{n}^{T}+\right.$ $\left.e_{n} \operatorname{diag}(\mathcal{T}(D))^{T}\right)$ and $\bar{S}=\bar{P}_{\pi}^{T} D_{1} \bar{P}_{\pi}=D-A=-4 \mathcal{T}(D)$. Hence, the nonzero eigenvalues of $\bar{S}=$ the nonzero eigenvalues of $-4 \mathcal{T}(D)=$ the nonzero eigenvalues of $-2 \mathcal{T}\left(D_{1}\right)$ since

$$
\mathcal{T}\left(D_{1}\right)=\left[\begin{array}{rr}
\mathcal{T}(D) & -\mathcal{T}(D) \\
-\mathcal{T}(D) & \mathcal{T}(D)
\end{array}\right]=\left[\begin{array}{rr}
1 & -1 \\
-1 & 1
\end{array}\right] \otimes \mathcal{T}(D),
$$

where $\otimes$ denotes the Kronecker product. Hence, $\chi_{\bar{S}}(\lambda)=\lambda^{n-r} \prod_{i=1}^{r}\left(\lambda+2 \mu_{i}\right)$, where $\mu_{i}, i=1, \ldots, r$, are the nonzero eigenvalues of $\mathcal{T}\left(D_{1}\right)$.

In order to establish (12), we still need to determine $\chi_{S}(\lambda)$. To this end, note that $\mathcal{T}\left(D_{1}\right) D_{1} e_{2 n}=2 n \mathcal{T}\left(D_{1}\right) \operatorname{diag}\left(\mathcal{T}\left(D_{1}\right)\right)=0$, where the first equality follows from (4). Then we have the following technical lemma.

Lemma 4.1. Let $D_{1}$ be an $2 n \times 2 n$ non-spherical EDM satisfying

$$
\mathcal{T}\left(D_{1}\right) D_{1} e_{2 n}=0
$$

Then

$$
D_{1} e_{2 n}=\frac{e_{2 n}^{T} D_{1} e_{2 n}}{2 n} e_{2 n}+\frac{2 n}{x_{1}^{T} x_{1}} x_{1},
$$

where $x_{1}$ satisfies (13). 
Proof. Let $\langle y\rangle$ denote the subspace generated by $y \in \mathfrak{R}^{2 n}$. Then it is shown in [1] that

$$
\text { Nullspace of } \mathcal{T}\left(D_{1}\right)=\left\langle e_{2 n}\right\rangle \oplus\left\langle x_{1}\right\rangle \oplus \text { Nullspace of } D_{1} \text {. }
$$

Thus,

$$
D_{1} e_{2 n}=\beta e_{2 n}+\gamma x_{1},
$$

for some scalars $\beta$ and $\gamma$. The result follows by multiplying equation (16) from the left with $e_{2 n}^{T}$ and $x_{1}^{T}$ respectively.

Therefore, it follows from (15) that $x_{1}^{T}=\left(x^{T} x^{T}\right)$ for some vector $x \in \mathfrak{R}^{n}$ and

$$
S e_{n}=(D+A) e_{n}=\frac{e_{2 n}^{T} D_{1} e_{2 n}}{2 n} e_{n}+\frac{2 n}{x_{1}^{T} x_{1}} x .
$$

Now, from the definition of $V_{n}$ in (10) we have $V_{n}^{T} S V_{n}=0$. Hence,

$$
\begin{aligned}
\chi_{S}(\lambda) & =\operatorname{det}\left(\lambda I_{n}-\left[\begin{array}{c}
V_{n}^{T} \\
\frac{e_{n}^{T}}{\sqrt{n}}
\end{array}\right] S\left[V_{n} \frac{e_{n}}{\sqrt{n}}\right]\right) \\
& =\operatorname{det}\left(\left[\begin{array}{cc}
\lambda I_{n-1} & -\frac{2 \sqrt{n}}{x_{1}^{T} x_{1}} V_{n}^{T} x \\
-\frac{2 \sqrt{n}}{x_{1}^{T} x_{1}} x^{T} V_{n} & \lambda-\frac{1}{2 n} e_{2 n}^{T} D_{1} e_{2 n}
\end{array}\right]\right) .
\end{aligned}
$$

Thus,

$$
\begin{aligned}
\chi_{S}(\lambda) & =\lambda^{n-2}\left(\lambda\left(\lambda-\frac{1}{2 n} e_{2 n}^{T} D_{1} e_{2 n}\right)-\frac{4 n}{\left(x_{1}^{T} x_{1}\right)^{2}} x^{T} V_{n} V_{n}^{T} x\right) \\
& =\lambda^{n-2}\left(\lambda\left(\lambda-\frac{1}{2 n} e_{2 n}^{T} D_{1} e_{2 n}\right)-\frac{2 n}{x_{1}^{T} x_{1}}\right),
\end{aligned}
$$

since $x^{T} V_{n} V_{n}^{T} x=x^{T} x=x_{1}^{T} x_{1} / 2$. Hence, (12) is established.

\subsection{Constructing cospectral EDMs}

Two EDMs are said to be cospectral if they have the same eigenvalues, i.e., if they have the same characteristic polynomial. In this subsection, we present a method for constructing two cospectral EDMs. 
Given a scalar $\mu>1$ and an $n \times n$ regular EDM $D$ generated by the points $p^{1}, \ldots, p^{n}$, let $D_{1}$ and $D_{2}$ be the two $2 n \times 2 n$ EDMs constructed from $D$ as follows. $D_{1}$ is generated by the $2 n$ points $p^{1}, p^{2}, \ldots, p^{n},-\mu p^{1},-\mu p^{2}, \ldots$, $-\mu p^{n}$; and $D_{2}$ is generated by the $2 n$ points $p^{1}, p^{2}, \ldots, p^{n}, \mu p^{1}, \mu p^{2}, \ldots$, $\mu p^{n}$. Next we show that $D_{1}$ and $D_{2}$ are cospectral.

Since $D$ is regular, we have $\operatorname{diag}(\mathcal{T}(D))=\frac{e_{n}^{T} D e_{n}}{2 n^{2}} e_{n}$. Thus it follows from (3) and (4) that

$$
D_{1}=\left[\begin{array}{cc}
D & A_{1} \\
A_{1} & \mu^{2} D
\end{array}\right], \quad \text { and } \quad D_{2}=\left[\begin{array}{cc}
D & A_{2} \\
A_{2} & \mu^{2} D
\end{array}\right]
$$

where

$$
A_{1}=\frac{1}{2 n^{2}} e_{n}^{T} D e_{n}\left(\mu^{2}+1\right) e_{n} e_{n}^{T}+2 \mu \mathcal{T}(D),
$$

and

$$
A_{2}=\frac{1}{2 n^{2}} e_{n}^{T} D e_{n}\left(\mu^{2}+1\right) e_{n} e_{n}^{T}-2 \mu \mathcal{T}(D)
$$

Note that $A_{1} e_{n}=A_{2} e_{n}=\frac{1}{2 n} e_{n}^{T} D e_{n}\left(\mu^{2}+1\right) e_{n}$. Therefore, the 2-partition $\pi$ of $D_{1}$ and $D_{2}$ corresponding to the normalized characteristic matrix

$$
P_{\pi}=\frac{1}{\sqrt{n}}\left[\begin{array}{cc}
e_{n} & 0 \\
0 & e_{n}
\end{array}\right]
$$

is equitable. Since

$$
\bar{P}_{\pi}=\left[\begin{array}{cc}
V_{n} & 0 \\
0 & V_{n}
\end{array}\right]
$$

it immediately follows that

$$
S_{1}=P_{\pi}^{T} D_{1} P_{\pi}=\frac{1}{n} e_{n}^{T} D e_{n}\left[\begin{array}{cc}
1 & \frac{1}{2}\left(\mu^{2}+1\right) \\
\frac{1}{2}\left(\mu^{2}+1\right) & \mu^{2}
\end{array}\right]=P_{\pi}^{T} D_{2} P_{\pi}=S_{2} .
$$

Furthermore,

$\bar{S}_{1}=\bar{P}_{\pi}^{T} D_{1} \bar{P}_{\pi}=\left[\begin{array}{cc}V_{n}^{T} D V_{n} & -\mu V_{n}^{T} D V_{n} \\ -\mu V_{n}^{T} D V_{n} & \mu^{2} V_{n}^{T} D V_{n}\end{array}\right]=\left[\begin{array}{cc}1 & -\mu \\ -\mu & \mu^{2}\end{array}\right] \otimes V_{n}^{T} D V_{n}$, 
and

$$
\bar{S}_{2}=\bar{P}_{\pi}^{T} D_{2} \bar{P}_{\pi}=\left[\begin{array}{cc}
V_{n}^{T} D V_{n} & \mu V_{n}^{T} D V_{n} \\
\mu V_{n}^{T} D V_{n} & \mu^{2} V_{n}^{T} D V_{n}
\end{array}\right]=\left[\begin{array}{cc}
1 & \mu \\
\mu & \mu^{2}
\end{array}\right] \otimes V_{n}^{T} D V_{n} .
$$

But the eigenvalues of $\bar{S}_{1}$ are equal to the eigenvalues of $\bar{S}_{2}$. In particular, the nonzero eigenvalues of $\bar{S}_{1}=$ the nonzero eigenvalues of $\bar{S}_{2}=\left(1+\mu^{2}\right)$ times the nonzero eigenvalues of $V_{n}^{T} D V_{n}=\left(1+\mu^{2}\right)$ times the nonzero eigenvalues of $-2 \mathcal{T}(D)$. Therefore, $D_{1}$ and $D_{2}$ are two cospectral EDMs. Furthermore, $D_{1}$ and $D_{2}$ are not regular since $\mu>1$.

\section{Constructing EDMs with three distinct eigenvalues}

EDMs have two or more distinct eigenvalues since the eigenvalues of an EDM $D$ can not all be equal. Moreover, EDMs with exactly two distinct eigenvalues are precisely those generated by the standard simplex, i.e., EDMs of the form $D=\gamma(E-I)$ for some scalar $\gamma>0$. The problem of obtaining a complete characterization of EDMs with exactly three distinct eigenvalues, just like its graph counterpart, seems to be difficult.

Neumaier [11] introduced the notion of strength for distance matrices as a measure of their regularity. According to Neumaier regular EDMs are of strength 1 while regular EDMs with three distinct eigenvalues, where one of the eigenvalues is a zero are of strength 2 . The following theorem follows from a more general result due to Neumaier [11].

Theorem 5.1. Let $D$ be a regular EDM with exactly three distinct eigenvalues such that its off-diagonal entries have exactly 2 or 3 distinct values. Then any two rows (columns) of $D$ are obtained from each other by a permutation.

Next we present two classes of non-regular EDMs with exactly 3 distinct eigenvalues. The first class consists of non-regular EDMs of order $n+1$ obtained from $n \times n$ regular EDMs with 3 distinct eigenvalues by adding one row and one column of equal entries.

Theorem 5.2. Let $D$ be an $n \times n$ regular EDM such that $\sigma(D)=\left\{e^{T} D e / n\right.$, $\left.-\lambda_{1}^{r_{1}},-\lambda_{2}^{n-1-r_{1}}\right\}$. Assume that either $\left(e^{T} D e / n+\lambda_{1}\right) \lambda_{1}=n \alpha^{2}$ or $\left(e^{T} D e / n+\right.$ 
$\left.\lambda_{2}\right) \lambda_{2}=n \alpha^{2}$ for some $\alpha \geq \frac{1}{2 n^{2}} e^{T} D e, \alpha \neq \frac{1}{n(n-1)} e^{T}$ De. Let $\gamma$ be some positive scalar. Then

$$
D_{1}=\gamma\left[\begin{array}{cc}
D & \alpha e_{n} \\
\alpha e_{n}^{T} & 0
\end{array}\right]
$$

is a non-regular EDM of order $n+1$ with exactly 3 distinct eigenvalues.

Proof. It is easy to see that $D_{1}$ is a non-regular EDM since $\alpha \neq \frac{1}{n(n-1)} e^{T} D e$. Now assume $\left(e^{T} D e / n+\lambda_{1}\right) \lambda_{1}=n \alpha^{2}$. Let $V$ be the $n \times(n-1)$ matrix whose columns form an orthonormal basis for subspace $M$ defined in (2), thus $V V^{T}=$ $J=I-e e^{T} / n$. Let

$$
Q=\left[\begin{array}{ccc}
e_{n} / \sqrt{n} & 0 & V \\
0 & 1 & 0
\end{array}\right]
$$

Then

$$
Q^{T} D_{1} Q=\gamma\left[\begin{array}{ccc}
e^{T} D e / n & \alpha n^{1 / 2} & 0 \\
\alpha n^{1 / 2} & 0 & 0 \\
0 & 0 & V^{T} D V
\end{array}\right] \text {. }
$$

But it is well known $[1,8]$ that $\sigma(-2 \mathcal{T}(D))=\sigma\left(V V^{T} D V V^{T}\right)=\left\{-\lambda_{1}^{r_{1}}\right.$, $\left.-\lambda_{2}^{n-1-r_{1}}, 0\right\}$. Thus $\sigma\left(V^{T} D V\right)=\left\{-\lambda_{1}^{r_{1}},-\lambda_{2}^{n-1-r_{1}}\right\}$. Furthermore, the submatrix

$$
\left[\begin{array}{cc}
e^{T} D e / n & \alpha n^{1 / 2} \\
\alpha n^{1 / 2} & 0
\end{array}\right]
$$

has eigenvalues $-\lambda_{1}$ and $\frac{1}{n} e^{T} D e+\lambda_{1}$. The result follows since $Q$ is an orthogonal matrix.

Note that, taking $\alpha=\frac{1}{2 n^{2}} e^{T} D e$ in Theorem 5.2 is equivalent to placing point $p^{n+1}$ at the center of the hyper-sphere containing $p^{1}, \ldots, p^{n}$. Also, note that if $\alpha=\frac{1}{n(n-1)} e^{T} D e$, then $D_{1}$ becomes a regular EDM.

The second class consists of non-regular EDMs of order $n+1$ obtained from EDMs of the form $D=\lambda\left(E_{n}-I_{n}\right), \lambda>0$, by adding one row and one column of equal entries. The proof of the next theorem is similar to that of Theorem 5.2.

Theorem 5.3. Let $\gamma$ be a positive scalar and $\alpha \geq(n-1) / 2 n, \alpha \neq 1$. Then

$$
D_{1}=\gamma\left[\begin{array}{cc}
E_{n}-I_{n} & \alpha e_{n} \\
\alpha e_{n}^{T} & 0
\end{array}\right]
$$


is a non-regular EDM with exactly 3 distinct eigenvalues, i.e., $\sigma\left(D_{1}\right)=$ $\left\{\lambda_{1},-\lambda_{2},-\gamma^{n-1}\right\}$ where

$$
\lambda_{1}=\frac{1}{2} \gamma\left(n-1+\sqrt{(n-1)^{2}+4 \alpha^{2} n}\right)
$$

and

$$
\lambda_{2}=\frac{1}{2} \gamma\left(n-1-\sqrt{(n-1)^{2}+4 \alpha^{2} n}\right) .
$$

Acknowledgement. The author would like to thank an anonymous referee for his/her comments and suggestions which improved the presentation of this paper.

\section{REFERENCES}

[1] A.Y. Alfakih, On the nullspace, the rangespace and the characteristic polynomial of Euclidean distance matrices. Linear Algebra Appl., 416 (2006), 348-354.

[2] G.M. Crippen and T.F. Havel, Distance Geometry and Molecular Conformation. Wiley, New York, 1988.

[3] F. Critchley, On certain linear mappings between inner-product and squared distance matrices. Linear Algebra Appl., 105 (1988), 91-107.

[4] C.D. Godsil, Compact graphs and equitable partitions. Linear Algebra Appl., 255 (1997), 259-266.

[5] C.D. Godsil and B.D. McKay, Feasibility conditions for the existence of walk-regular graphs. Linear Algebra Appl., 30 (1980), 51-61.

[6] J.C. Gower, Properties of Euclidean and non-Euclidean distance matrices. Linear Algebra Appl., 67 (1985), 81-97.

[7] T.L. Hayden, J. Lee, J. Wells and P. Tarazaga, Block matrices and multispherical structure of distance matrices. Linear Algebra Appl., 247 (1996), 203-216.

[8] T.L. Hayden and P. Tarazaga, Distance matrices and regular figures. Linear Algebra Appl., 195 (1993), 9-16.

[9] J. De Leeuw and W. Heiser, Theory of multidimensional scaling. In: P.R. Krishnaiah and L.N. Kanal (Eds.), Handbook of Statistics, volume 2, pages 285-316. North-Holland (1982).

[10] A. Mowshowitz, The adjacency matrix and the group of a graph. In: F. Harary (Ed.), New directions in the theory of graphs, pages 129-148. Academic Press, New York (1973).

[11] A. Neumaier, Distances, graphs and designs. Europ. J. Combinatorics, 1 (1980), 163-174.

[12] A. Neumaier, Distance matrices, dimension and conference graphs. Nederl. Akad. Wetensch. Indag. Math., 43 (1981), 385-391. 
[13] H. Sachs, Über teiler, faktoren und charakteristische polynome von graphen. Teil I. Wiss. Z. TH Ilmenau, 12 (1966), 7-12.

[14] I.J. Schoenberg, Remarks to Maurice Fréchet's article: Sur la définition axiomatique d'une classe d'espaces vectoriels distanciés applicables vectoriellement sur l'espace de Hilbert. Ann. Math., 36 (1935), 724-732.

[15] A.J. Schwenk, Computing the characteristic polynomial of a graph. In Graphs and Combinatorics, Lecture Notes in Mathematics, Vol. 406, pages 153-162. Springer, Berlin (1974).

[16] A. M-C So and Y. Ye, Theory of semidefinite programming for sensor network localization. Math. Program. Ser. B, pages 367-384, 2007.

[17] P. Tarazaga, T.L. Hayden and J. Wells, Circum-Euclidean distance matrices and faces. Linear Algebra Appl., 232 (1996), 77-96.

[18] G. Young and A.S. Householder, Discussion of a set of points in terms of their mutual distances. Psychometrika, 3 (1938), 19-22. 